\title{
Analysis of cystic fibrosis-associated P67L CFTR illustrates barriers to personalized therapeutics for orphan diseases
}

\author{
Carleen M. Sabusap, ${ }^{1}$ Wei Wang, ${ }^{1}$ Carmel M. McNicholas, ${ }^{1}$ W. Joon Chung, ${ }^{1}$ Lianwu Fu, ${ }^{1}$ Hui Wen, \\ Marina Mazur, ${ }^{1}$ Kevin L. Kirk, ${ }^{1}$ James F. Collawn, ${ }^{1}$ Jeong S. Hong, ${ }^{1}$ and Eric J. Sorscher ${ }^{1,2}$ \\ ${ }^{1}$ Gregory Fleming James Cystic Fibrosis Research Center, University of Alabama, Birmingham, Alabama, USA. \\ ${ }^{2}$ Emory University, Atlanta, Georgia, USA.
}

\begin{abstract}
Emerging knowledge indicates the difficulty in categorizing unusual cystic fibrosis (CF) mutations, with regard to both pathogenic mechanism and theratype. As case in point, we present data concerning P67L mutation of the cystic fibrosis transmembrane conductance regulator (CFTR), a defect carried by a small number of individuals with CF and sometimes attributed to a channel conductance abnormality. Findings from our laboratory and others establish that P67L causes protein misfolding, disrupts maturation, confers gating defects, is thermally stable, and exhibits near normal conductance. These results provide one framework by which rare CF alleles such as P67L can be more comprehensively profiled vis-à-vis molecular pathogenesis. We also demonstrate that emerging CF treatments - ivacaftor and lumacaftor - can mediate pronounced pharmacologic activation of P67L CFTR. Infrequent CF alleles are often improperly characterized, in part, due to the small numbers of patients involved. Moreover, access to new personalized treatments among patients with ultra-orphan genotypes has been limited by difficulty arranging phase III clinical trials, and off-label prescribing has been impaired by high drug cost and difficulty arranging third party reimbursement. Rare CFTR mutations such as P67L are emblematic of the challenges to "precision" medicine, including use of the best available mechanistic knowledge to treat patients with unusual forms of disease.
\end{abstract}

Conflict of interest: E.J. Sorscher is a nonvoting member of the Cystic Fibrosis Foundation Board of Trustees.

Submitted: January 19, 2016 Accepted: July 21, 2016 Published: September 8, 2016

Reference information: JCI Insight. 2016;1(14):e86581. doi:10.1172/jci.insight.86581.

\section{Introduction}

Mutations of the cystic fibrosis transmembrane conductance regulator (CFTR) lead to damage of respiratory, hepatic, pancreatic, and other exocrine tissues (1-3). CFTR functions as a chloride and bicarbonate ion channel, for which over 1,900 disease-associated mutations have been implicated. These defects are predominately classified into 1 of 6 groups, according to underlying molecular mechanism (abnormalities of CFTR ion channel gating, folding, premature truncation, aberrant splicing, etc.). Significant limitations of the current CFTR classification scheme have become increasingly evident. For example, approximately $90 \%$ of cystic fibrosis (CF) patients carry at least one copy of the prevalent F508del allele, which is classically associated with a protein biogenesis (class II) defect (3-5). More detailed analysis has established that F508del mediates disease through not only aberrant protein maturation, but also dysfunctional gating (class III) and plasma membrane instability (class VI) (6, 7). Mutations less common than F508del are poorly characterized from a mechanistic standpoint but in the aggregate represent a very large number of individuals with CF.

The P67L mutation results from a proline-to-leucine replacement at position 67 of the 1,480-amino acid CFTR. The defect was first described as producing a mild clinical phenotype and occurs at high frequency among individuals of Scottish descent (8). Patients carrying P67L are often pancreatic sufficient and may be diagnosed later in life; many exhibit comparatively moderate abnormalities of forced expiratory volume in 1 second (FEV1) and sweat chloride (8). Designation of P67L as a "conductance" or class IV defect (9) implies structural abnormalities of the CFTR transmembrane pore. It might be argued on mechanistic grounds that class IV mutations would not respond to clinically approved cell processing correctors such as lumacaftor. Moreover, class IV assignment of P67L has constituted a basis for clinical decision making, the pharmaceutical approach to FDA registration, and third party reimbursement for recently approved CFTR 
A

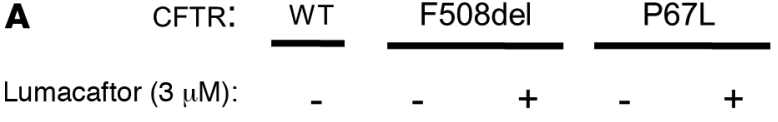

CFTR (10B6.2)

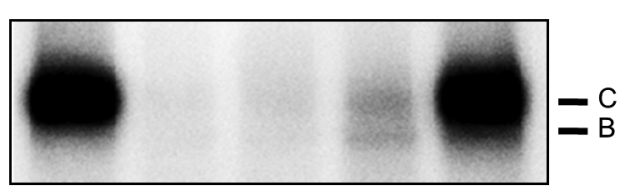

$\beta$-actin
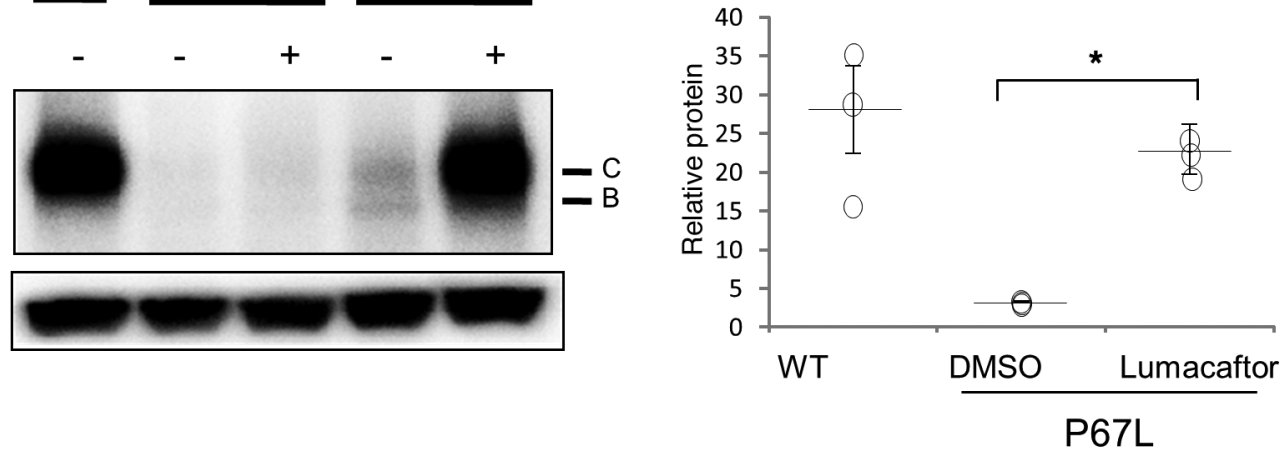

B
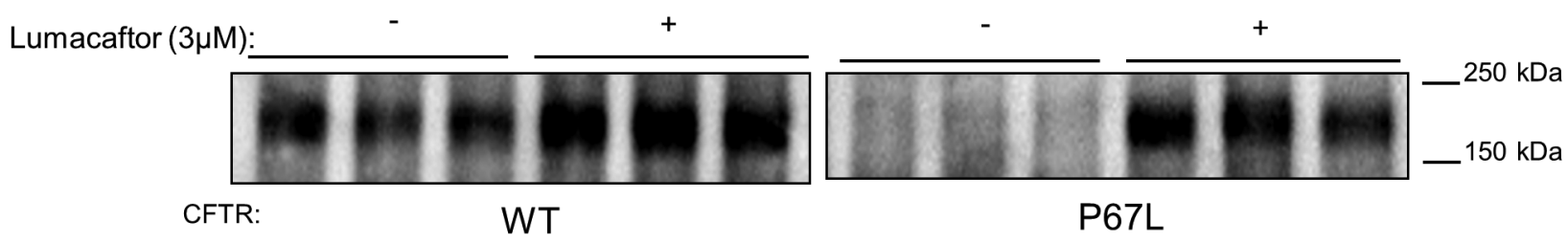

Figure 1. The P67L mutant confers reduced expression of mature cystic fibrosis transmembrane conductance regulator (CFTR). (A) Fischer rat thyroid (FRT) cells stably expressing WT, F508del mutant, or P67L mutant CFTR from an identical genomic integration site were treated with $3 \mu \mathrm{M}$ lumacaftor. After 24 hours, cells were lysed, and total cellular protein was analyzed by Western blotting with anti-human CFTR antibodies (10B6.2). Decreased postER compartment, fully glycosylated (band C) CFTR was observed in the P67L sample compared with that in the WT sample and was efficiently rescued by lumacaftor ( $n=3$ technical replicates, ${ }^{*} P=0.0008$ via 2-sample $t$ test) (ER-localized, core-glycosylated CFTR is designated as B). The baseline defect observed for untreated P67L CFTR shown here is consistent with previous biochemical observations for this mutant (27). Data are mean \pm SEM; some error bars are hidden by symbols. (B) In FRT cells biotinylated to detect surface CFTR, incubation with $3 \mu \mathrm{M}$ lumacaftor increased plasma membrane abundance. This experiment was repeated with similar results.

modulators. The mechanistic category, therefore, can have far-reaching implications, and it is emblematic of the situation among many CF mutant alleles, for which precision treatments are being contemplated.

In the present study, we provide evidence that P67L CFTR exhibits deficient biogenesis and substantial channel gating abnormalities distinct from a previously assigned conductance defect. This includes a deficit in open probability and normal conduction as well as diminished maturation/trafficking. Furthermore, we show that P67L-related aberrations can be overcome using the FDA-approved CFTR modulators, ivacaftor (Kalydeco) and lumacaftor. Our findings illustrate the limitations of CF molecular phenotype as the primary determinant of therapeutic access. In $\mathrm{CF}$ and other conditions for which caregivers possess molecular and clinical tools to rationally evaluate a personalized intervention, significant challenges to achieving the goal of therapeutic "precision" remain.

\section{Results}

Biogenesis of P67L. Levels of mature, fully glycosylated P67L CFTR were markedly reduced compared with those of WT CFTR but were higher than those of F508del CFTR, which required longer exposure times for detection (Figure 1A). To further evaluate protein biogenesis, cells were incubated with lumacaftor, a CFTR modulator recently approved for clinical use. Protein expression of the mature, fully glycosylated P67L band C increased dramatically — with levels comparable to those of WT CFTR — following lumacaftor administration. This increase was tested further using biotinylation to verify P67L band C at the cell surface (Figure 1B) $(10,11)$. The data indicate that $\mathrm{P} 67 \mathrm{~L}$ is significantly misprocessed and suggest that the P67L pathogenic mechanism is, at least in part, a class II defect. Related biogenesis abnormalities (nucleotide binding domain 1 [NBD1] misfolding, defective cytosolic loop/NBD1 binding, plasma membrane instability [a class VI defect]) may also contribute to the findings shown here $(4,5,11)$.

Functional P67L rescue by ivacaftor and lumacaftor. The capacity of ivacaftor and lumacaftor to restore activity of P67L CFTR stably expressed in polarized Fischer rat thyroid (FRT) cells and primary nasal airway epithelial cells was examined by Ussing chamber analysis. Short-circuit current $\left(\mathrm{I}_{\mathrm{sc}}\right)$ following treatment with these compounds led to enhancement of CFTR-mediated chloride transport in cells expressing P67L or F508del CFTR (Figure 2, A and B). Primary airway epithelial cells encoding a P67L/F508del 

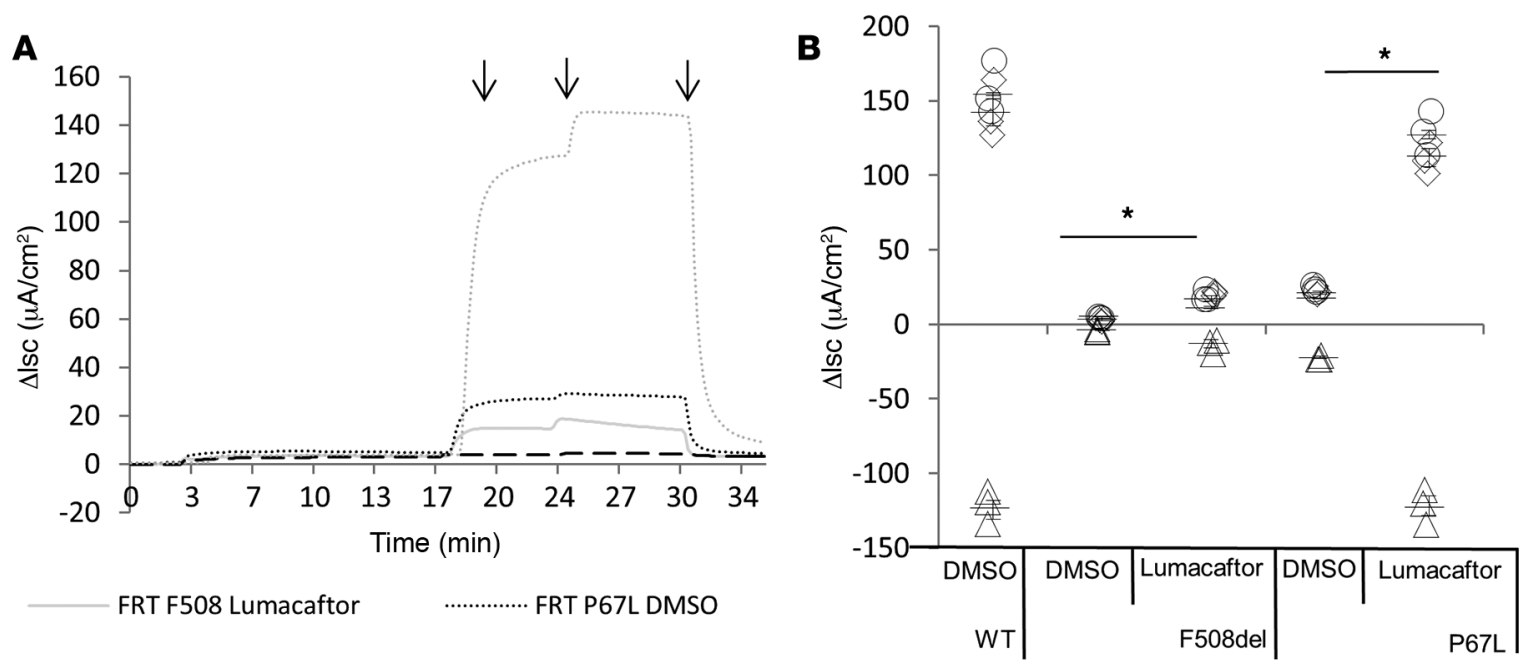

- - FRT F508 DMSO

FRT P67L Lumacaftor

C

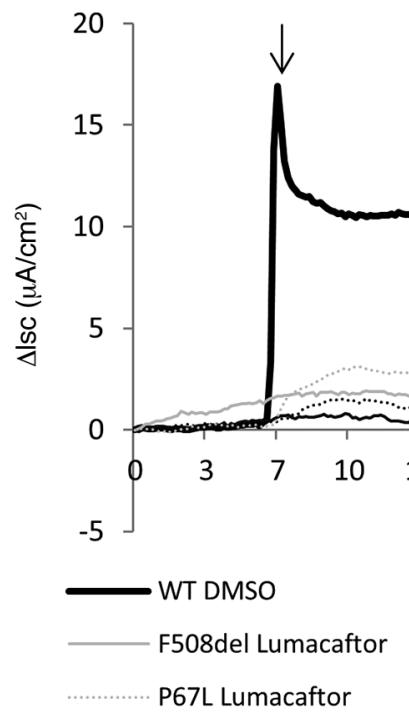

E

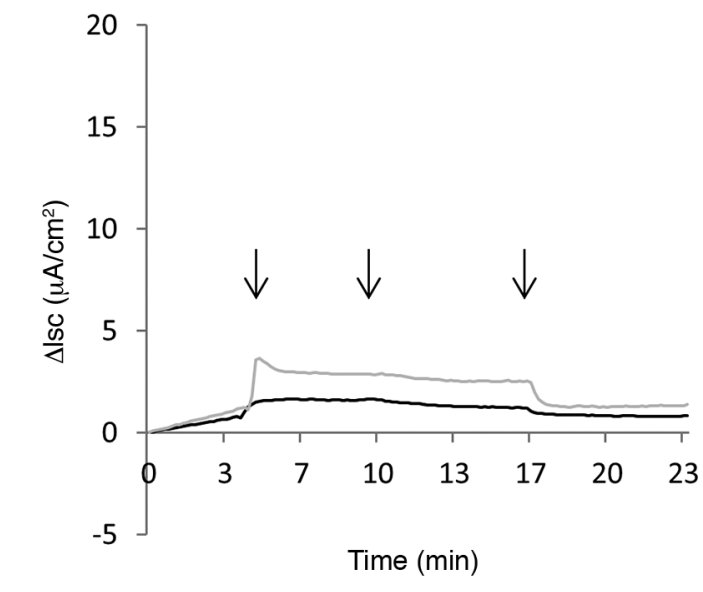

—F508del DMSO — F508del Lumacaftor
D 15

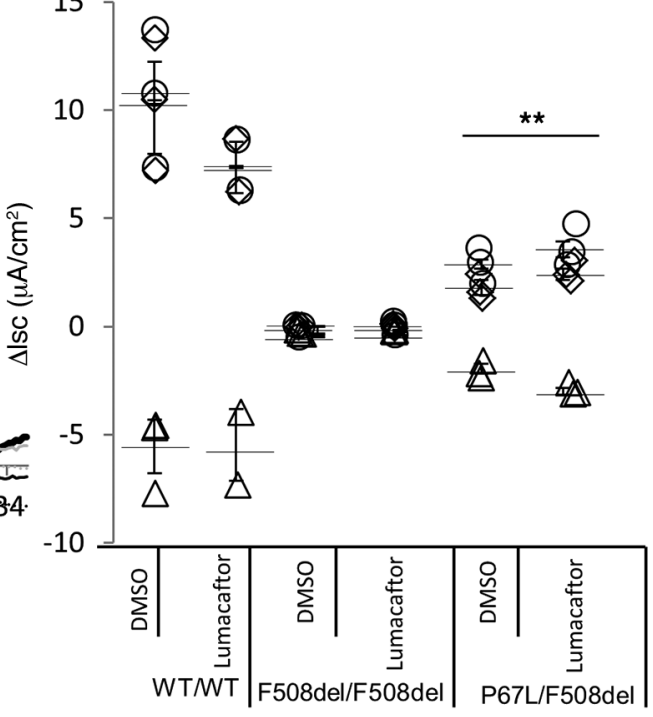

$\mathbf{F}$

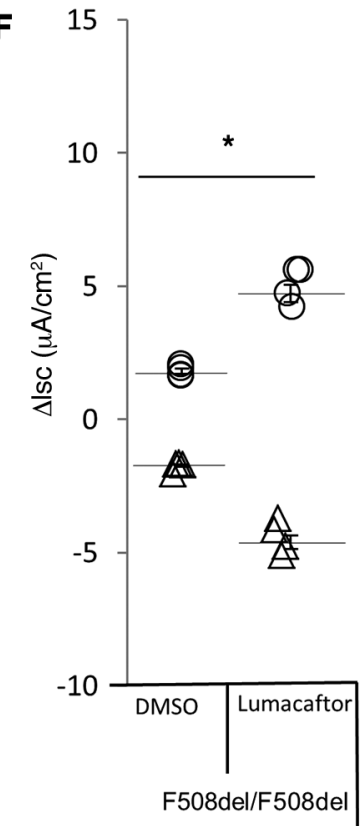


Figure 2. Functional assessment of the P67L cystic fibrosis transmembrane regulator (CFTR) mutant in polarized Fischer rat thyroid (FRT) and primary airway epithelial cells. Ussing chamber experiments were carried out in FRT (A and B) or primary airway cells from 3 cystic fibrosis patients (genotypes P67L/F508del, F508del/F508del, F508del/F508del; C-F) at $37^{\circ} \mathrm{C}$ following 24 hours of treatment with DMSO (control) or $3 \mu \mathrm{M}$ lumacaftor. Data showing short-circuit current (Isc) after luminal stimulation with forskolin ( $10 \mu \mathrm{M}$, far left arrows) and ivacaftor ( $300 \mathrm{nM}$, middle arrows) and inhibition by CFTR inh172 (30 $\mu \mathrm{M}$, a CFTR inhibitor, far right arrows) are depicted in A, C, and E. Summary data is shown as mean \pm SEM in B, D, and F (diamonds, forskolin; circles, ivacaftor + forskolin; triangles, inh172); some error bars are hidden by symbols. Variable response to lumacaftor is characteristic of the two independent homozygous F508del CFTR mutant patient codes shown in C-F. Dot plots describe $n=3-4$ filters (technical replicates for each cell line or patient sample) per condition ( $n=2$ for lumacaftor-treated WT/WT primary cells). ${ }^{*} P<0.05$ for forskolin + ivacaftor stimulation; ${ }^{* *} P=0.02$ for inh172 inhibition (indicative of total constitutive + activated CFTR function), determined via 2-sample $t$ test.

CFTR genotype exhibited robust activity at baseline and following treatment with ivacaftor and forskolin, approximating 40\%-50\% of WT (Figure 2, C and D). Variable effects were observed for distinct F508del homozygous patient codes (Figure 2, C-F), consistent with the observation that certain patient samples tested in this fashion may exhibit minimal responsiveness.

Examination of P67L channel activity. Gating of WT and P67L CFTR in FRT and primary nasal epithelial cells was examined in the context of single and multiple channels using inside-out cell patch analyses. Increased open probability was observed for both FRT WT and P67L CFTR following treatment with ivacaftor (Figure 3, A-C). A current/voltage relationship resembling that for WT CFTR was observed for P67L CFTR in FRT cells (Figure 3, D and E). A similar result was obtained in P67L/F508del primary cells (Figure 4). Based on the established observation that a negligible amount of F508del CFTR is present at the cell surface in primary airway epithelia $(12,13)$ and that P67L/F508del airway samples exhibit strong CFTR localization at the epithelial surface in vitro (Figure 2, C and D) and in vivo (9), these findings further indicate that P67L CFTR encodes a normal chloride conductive pore.

Thermostability of P67L ion channels. Figure 5 illustrates the effect of warming the bath to physiological temperature during P67L CFTR recording in excised, inside-out membrane patches. It has been shown previously that WT CFTR maintains stable current at physiological temperature, whereas F508del CFTR rapidly and irreversibly loses activity under the same conditions (14). We studied the influence of temperature on lumacaftor-rescued P67L CFTR in this context. Macroscopic currents from P67L CFTR increased in response to elevated temperature (physiological) and reversibly returned to control levels upon cooling the bath (Figure 5A) (14). Figure 5B shows the stability of lumacaftor-rescued P67L CFTR at $37^{\circ} \mathrm{C}$.

\section{Discussion}

Despite an advanced understanding of causative alleles in CF, establishment of precise molecular intervention remains problematic $(5-7,15)$. For example, comparatively straightforward pathogenic categories (gating, trafficking, conductance, etc.) have allowed CF defects to be classified in a fashion intended to predict clinical response by tailored or precision treatments (16). However, even for well-described CFTR abnormalities, such as F508del, personalized therapies must also account for non-CFTR gene modifiers, epigenetics, CFTR allelic polymorphism (17), somatic cell chimerism, and environmental exposures among other features. Any of these might influence clinical phenotype and/or therapeutic effect. Importantly, among patients with ultra-orphan alleles, identification of a causal (and fundamental) mechanism does not necessarily mean a specific patient will benefit from a precise treatment.

The complexity of this perspective and its relevance to personalized medicine is placed in relief by P67L CFTR, a mutation emblematic of hundreds of other rare CF alleles. First, P67L has sometimes been incorrectly grouped as a class IV (conduction) defect, perhaps based, in part, on decreased CFTR currents observed previously for a different mutation at the same position, P67S (18). The categorization as class IV argues against usefulness of ivacaftor or lumacaftor and might otherwise be taken as evidence to deter pharmaceutical trials seeking FDA approval and/or third party reimbursement with regard to available CFTR modulators. In addition, for patients suffering from an orphan disease - CF being one example - a limited market often leads to specialized but very expensive therapeutic agents. Although third party reimbursement may require FDA approval for the diagnosed indication, an insufficient number of subjects for clinical trial evaluation impedes the conventional drug approval process. Such barriers have been highlighted previously from an ethical perspective (19-22), and specific examples also include access to anticancer and anti-infective treatments $(20,23,24)$.

Second, data from our laboratory and others indicate robust activity of clinically approved CFTR modulators for P67L CFTR in multiple cell systems and in vivo (Figures 1-3) $(9,12,25)$. The present studies show 

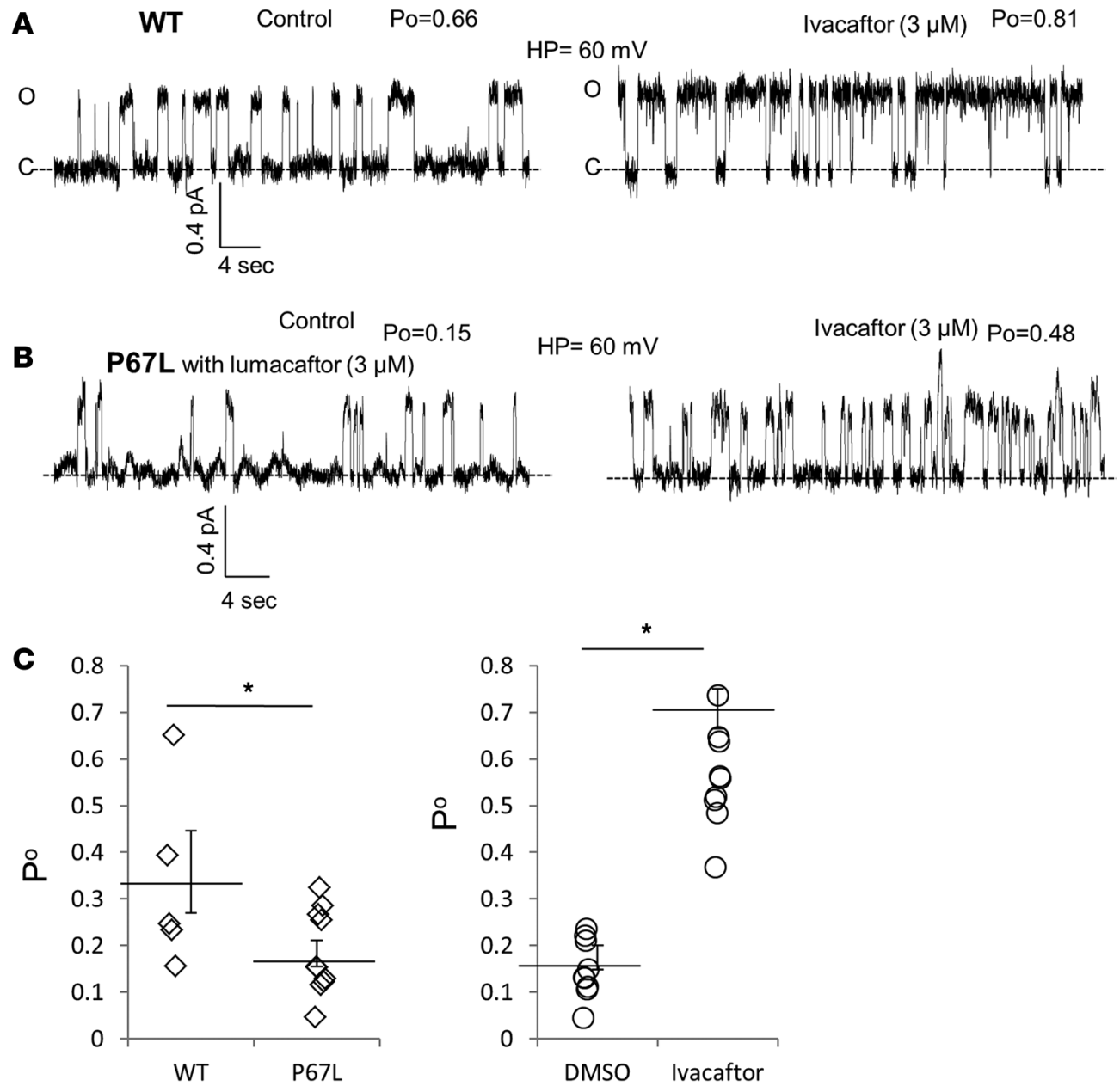

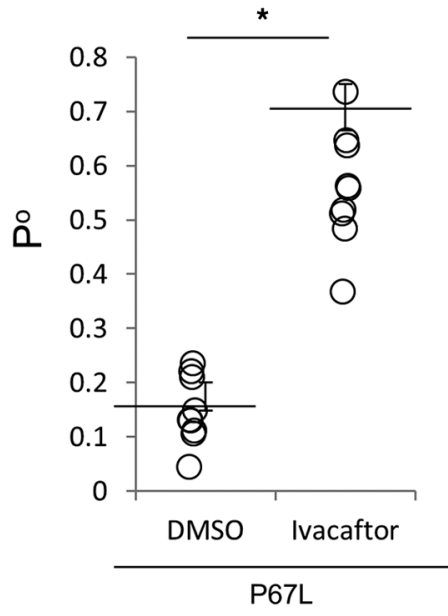

D

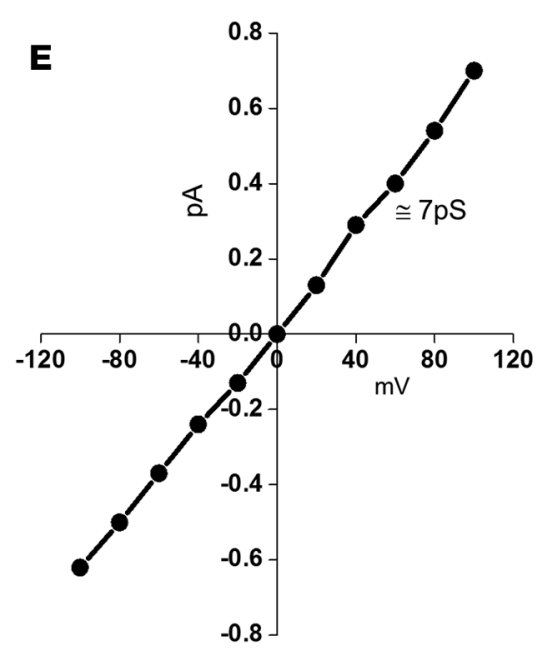

Figure 3. Effect of ivacaftor on single-channel characteristics in Fischer rat thyroid (FRT) cells. (A) WT cystic fibrosis transmembrane regulator (CFTR). (B) Lumacaftor-treated P67L mutant CFTR. HP, holding potential; $P_{0}$, open probability; O, open; C, closed configuration. Summary data are shown in $\mathbf{C}$ as mean $\pm \mathrm{SEM}$, $n=5-10$ experiments (technical replicates) per condition, ${ }^{*} P<$ 0.05 ( $P=0.03$, left panel; $P=1.2$

$\times 10^{-8}$, right panel) via 2 -sample $t$ test. Tracings with multiple WT or P67L CFTR channels in FRT cells are shown in D. (E) P67L exhibits a linear current/voltage relationship of approximately 7 picosiemens (pS), characteristic of a properly conducting CFTR channel. This experiment was conducted with $n=3$ CFTR channel technical replicates.

that the class IV designation for $\mathrm{P} 67 \mathrm{~L}$ is incorrect and that the mutation causes protein misfolding, impairs biogenesis, confers gating defects, and exhibits normal conductance. Considerations such as these illustrate the importance of thorough evaluation for any rare allele in terms of molecular pathogenesis and theratype. Based on emerging knowledge regarding more common CFTR defects, it is likely that hundreds of CFTR mutations have been placed in categories that are grossly oversimplified, i.e., a single mutation eliciting a single defect in CFTR appears to be an exception. This is particularly the case when one considers that even premature truncation alleles may be improved by lumacaftor or ivacaftor (26). The present results provide one means by which a more complete molecular profile for rare $\mathrm{CF}$ alleles can be obtained in the future.

Third, under the current pharmaceutical testing paradigm and third party reimbursement policies, patients with P67L (like many other rare CFTR mutations) are unlikely to be prescribed drugs such as ivacaftor or Orkambi (an FDA-approved treatment containing ivacaftor and lumacaftor). Notably, the preclinical results in Figures 1-5 are supported by observations in a patient given ivacaftor (9). From this perspective, one can argue that, for rare and poorly described mutations, novel approaches to the complex issue of drug registration should be entertained. For example, based on a need to thoroughly characterize 
A

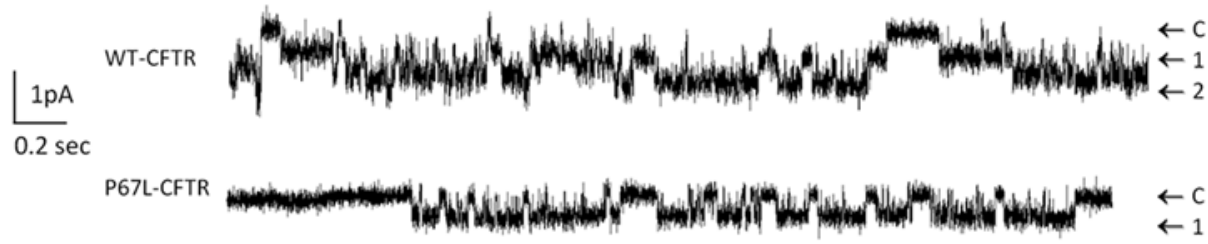

B

WT (7.6 pS)

- P67L (8.1 pS)

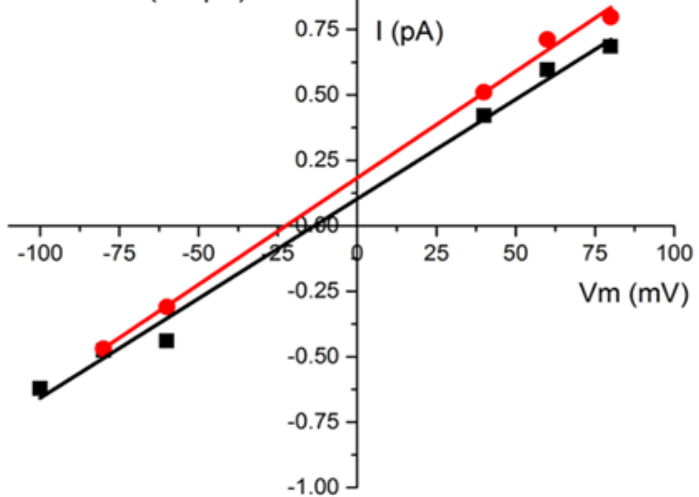

Figure 4. Cystic fibrosis transmembrane regulator (CFTR) channels detected in primary WT or mutant P67L/F508del airway cells. (A) Single-channel trace. (B) Current (I)/voltage relationship for P67L appears very similar to that for WT. C, closed; 1 and 2, number of open channels. This experiment and analysis were conducted with $n=3$ WT and $n=2$ P67L CFTR channel technical replicates. $\mathrm{Vm}$, transmembrane voltage.

"personalized" interventions for unusual CF defects, development of innovative preclinical model systems that predict patient responsiveness could represent a valuable adjunct during the drug registration process for orphan CF alleles. Moreover, in the same manner that antibiotics, analgesics, chemotherapy, antihypertensives, and most drug therapies are commonly administered and monitored for clinical effect, formal mechanisms that allow physicians (within FDA-specified guidelines) to prescribe CF modulators might be considered. Given the difficulty arranging phase III clinical studies for very small CF patient sub-groups, innovative protocols designed to evaluate efficacy could help improve access among patients with rare alleles. In this context, theoretically, if FDA permission could be obtained, a 3- to 4-week clinical trial that shows improved FEV1 might justify continued treatment using a CFTR modulator. Establishing meaningful thresholds for provisional drug administration is not trivial, and patient eligibility for such a proposal would obviously require compelling evidence of safety in human subjects and strong data in support of genotype-specific effectiveness. That being said, formalizing access mechanisms of this sort could allow establishment of rigorous phase IV clinical trials to confirm patient benefit and a more systematic evaluation of emerging precision interventions. In either case, arrangements such as these would empower physicians in the clinic to offer the best available therapies, facilitate third party reimbursement, and favor patients who — at present — have inadequate access to potentially effective treatments.

The findings presented here are concordant with those in earlier studies. For example, Kraus et al. noted diminished ion transport attributable to P67L. Based, in part, on abnormal current/voltage relationship in the closely related P67S defect, P67L may have been incorrectly assigned class IV status (18). Other data from large surveys of rare $\mathrm{CF}$ alleles also point to significant processing and/or transport abnormalities associated with P67L $(12,25,27)$. These previous reports furnish additional support for complexity of the molecular mechanism underlying P67L CFTR and reinforce the analysis described above.

In summary, patients with rare CFTR mutations such as P67L often cannot gain access to leading edge $\mathrm{CF}$ compounds unless they possess an FDA-approved genotype, and third party payers have struggled with the complexity of providing reimbursement for individuals carrying uncommon alleles until FDA registration is available. The biochemical, monolayer, and single-channel findings shown here indicate that individuals with P67L CFTR could potentially benefit from ivacaftor or Orkambi, if in vivo confirmation and regulatory approval were in place. Thorough evaluation of rare CFTR alleles, together with an approach that allows physicians more opportunity to evaluate and treat patients based on well-validated preclinical or clinical endpoints and FDA approval, would serve many individuals with rare forms of the disease. 


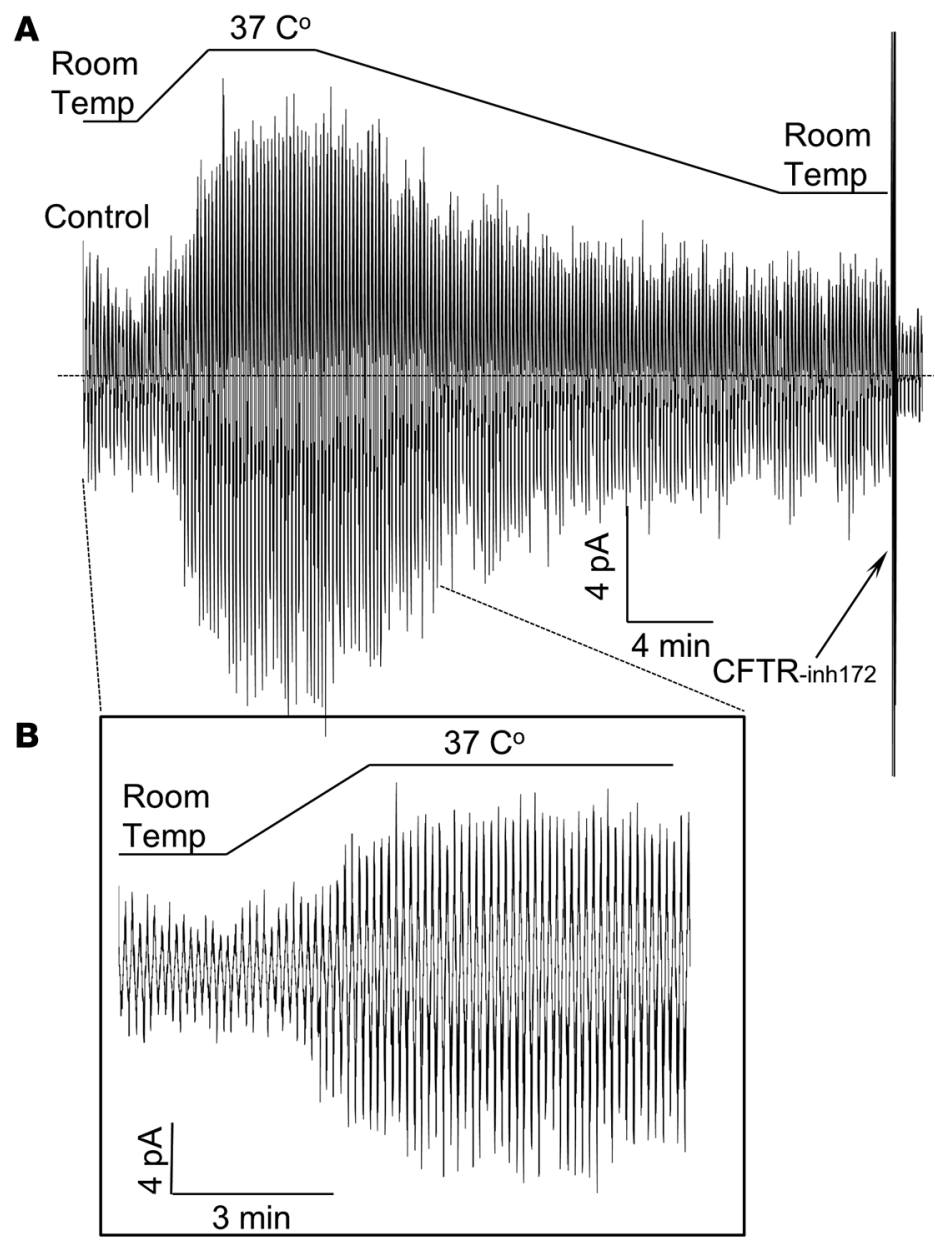

Figure 5. Thermal stability of mutant P67L cystic fibrosis transmembrane conductance regulator (CFTR) following lumacaftor rescue. Macroscopic currents obtained using inside-out excised patches were evoked by a ramp protocol from -80 to $+80 \mathrm{mV}$. Membrane patches were exposed to ATP (1.5 mM) and PKA (110 U/ $\mathrm{ml}$ ) in the bath at room temperature (control conditions). (A) P67L currents shown for an extended period with temperature cooling. (B) P67L CFTR during elevation of the bath to $37^{\circ} \mathrm{C}$. In contrast to the CFTR mutant F508del (14), lumacaftor-rescued P67L CFTR channels remain active at physiological temperature. This experiment has been repeated with similar results. inh172, an inhibitor of CFTR.

\section{Methods}

FRT cell line generation. FRT cells were provided by M. Welsh (University of Iowa, Iowa City, Iowa, USA). Cells were modified by our laboratory to express WT or mutant CFTR on an isogenic background using the Flp-in system (Thermo Fisher). Briefly, CFTR cDNAs (WT, F508del, P67L) were cloned into NheI and XhoI sites of the pcDNA5/FRT expression vector according to the manufacturer's instructions (Thermo Scientific). Cells were cotransfected with plasmids containing CFTR and a flp recombinase construct (pOG44) and cultured in media with hygromycin $(100 \mu \mathrm{g} / \mathrm{ml})$ to select site-specific recombinants. Hygromycin-resistant clonal lines were isolated and screened for loss of $\beta$-galactosidase. Clonal cells were found to have CFTR mRNA levels that varied less than by $50 \%$ compared with cells expressing WT CFTR, in contrast with more variable levels of mRNA in FRT lines tested previously (25). Stability of expression in the new FRT models was verified to at least passage 31. All new lines were validated by Western blot and $\mathrm{I}_{\mathrm{sc}}$ measurements with and without lumacaftor or ivacaftor. The P67L biogenesis defect and responses to lumacaftor were also shown following transient transfection in human embryonic kidney (HEK) 293 cells (ATCC, catalog CRL-3216; E.J. Sorscher, unpublished observations).

Cell culture. FRT cells were maintained in Coon's modified Ham's F12 (Sigma-Aldrich), supplemented with $5 \%$ fetal bovine serum and grown at $37^{\circ} \mathrm{C}$ in a humidified incubator containing $5 \% \mathrm{CO}_{2} / 95 \% \mathrm{O}_{2}$. Primary human nasal epithelial cells were collected from non-CF, homozygous F508del, and P67L/F508del individuals. Lower airway epithelial F508del/F508del monolayers were also evaluated. At 80\%-90\% confluency, primary cells were seeded onto $6.5 \mathrm{ml}$ permeable supports $\left(\sim 0.5 \times 10^{6}\right.$ cells per filter; Corning) and coated with NIH 3 T3 fibroblast-conditioned (ATCC, catalog CRL-1658) media. Cells were then propagated in differentiation media containing DMEM/F12 (Invitrogen), 2\% Ultroser G (Pall), 2\% Fetal Clone (Hyclone), $2.5 \mu \mathrm{g} / \mathrm{ml}$ insulin (Sigma-Aldrich), and $0.25 \%$ bovine brain extract (Lonza). $20 \mathrm{nM}$ hydrocortisone, $500 \mathrm{nM}$ triiodothyronine, $2.5 \mu \mathrm{g} / \mathrm{ml}$ transferrin (Invitrogen), $250 \mathrm{nM}$ ethanolamine, $1.5 \mu \mathrm{M}$ epinephrine, $250 \mathrm{nM}$ phosphoethanolamine, and $10 \mathrm{nM}$ retinoid acid were included in the media until terminally differentiated ( $\sim 4$ weeks). For rescue and delivery of F508del and P67L CFTR to the plasma membrane, cells were incubated with $3 \mu \mathrm{M}$ lumacaftor (Selleck Chemicals) for 24 hours.

Antibodies and chemicals. Anti-CFTR mouse monoclonal antibodies included 10B6.2 anti-human NBD1 monoclonal (http://www.cftrfolding.org/reagentRequests10B62.asp), UNC570 R domain (recognizes CFTR aa 731-742, code no. A2), 596 NBD2 (aa 1204-1211, code no. A4), and 24-1 anti-C-terminus monoclonal antibodies (http://www.unc.edu/ tjjensen/CFADP/) (all provided by J. Riordan, University of North Carolina, Chapel Hill, North Carolina, USA).

Polyclonal anti- $\beta$-actin antibody was purchased from Sigma-Aldrich (catalog A5441). HRP-conjugated goat anti-mouse IgG and anti-rabbit IgG were obtained from Bio-Rad (1706516 and 1721034, respectively). SuperSignal West Pico chemiluminescence substrate and CFTR potentiator (ivacaftor) were from Thermo Forma. All other chemicals were purchased from Sigma-Aldrich, Thermo Scientific, or as described below. 
Western blotting. Cells were washed with PBS, pelleted, and lysed with radioimmunoprecipitation buffer containing protease inhibitor cocktail and EDTA for 45 minutes on ice. Protein concentration was measured using a Bicinchoninic acid assay (Thermo Scientific). Briefly, $40 \mu \mathrm{g}$ cell lysate from each sample was resolved by SDS-PAGE and transferred to PVDF membranes, followed by blotting with specific primary antibody and HRP-labeled secondary antibody. The membrane was developed using chemiluminescent substrate (SuperSignal West Pico, Thermo Scientific) and signal was quantified by a ChemiDoc XRS System (Bio-Rad). Densitometry was performed using ImageJ software (NIH).

Cell surface biotinylation. The cell surface expression of P67L and F508del CFTR was measured after biotin labeling using EZ-link Biotin-LC-hydrazide as described previously (10). Following surface biotinylation of cells, P67L and F508del CFTR were immunoprecipitated using the C-terminal CFTR antibody (24-1, R\&D Systems) and then blotted with Avidin D-HRP (10).

Ussing chamber analyses. $\mathrm{I}_{\mathrm{sc}}$ was measured under voltage clamp conditions with an MC8 instrument and P2300 Ussing chamber equipment (Physiologic Instruments) (28). Human primary epithelial and FRT CFTR monolayers were bathed on both sides with identical Ringer's solutions containing the following (in $\mathrm{mM}$ ): $115 \mathrm{NaCl}, 25 \mathrm{NaHCO}_{3}, 2.4 \mathrm{KH}_{2} \mathrm{PO} 4,1.24 \mathrm{~K}_{2} \mathrm{HPO}_{4}, 1.2 \mathrm{CaCl}_{2}, 1.2 \mathrm{MgCl}_{2}$, and $10 \mathrm{D}$-glucose (pH 7.4). Solutions were vigorously stirred by bubbling through $5 \% \mathrm{CO}_{2} / 95 \% \mathrm{O}_{2}$. One-second 3-mV pulses were imposed every 10 seconds to calculate resistance by Ohm's law. Where indicated, mucosal solutions were changed to a low $\mathrm{Cl}^{-}$buffer $\left(1.2 \mathrm{mM} \mathrm{NaCl}\right.$ and $115 \mathrm{mM} \mathrm{Na}^{+}$gluconate, with other components as above). Amiloride $(100 \mu \mathrm{M})$ was added to block residual $\mathrm{Na}^{+}$current, followed by the CFTR agonist forskolin $(20$ $\mu \mathrm{M})$ and potentiator ivacaftor $(300 \mathrm{nM})$. $\operatorname{Inh}_{172}(10 \mu \mathrm{M})$, an inhibitor of CFTR, was added to the apical solution at the completion of each experiment to block CFTR-dependent $\mathrm{I}_{\mathrm{sc}}$. All chambers were maintained at $37^{\circ} \mathrm{C}$ during current recording.

Single-channel activity. FRT cells or primary nasal epithelial cells expressing CFTR (from non-CF or P67L/F508del individuals) were seeded on glass coverslips and mounted in flow-through chambers. Single-channel currents were recorded from inside-out patches using the Molecular Devices Axopatch 200B/ pClamp 10 system (29). Currents were obtained under symmetrical $\mathrm{Cl}^{-}$at room temperature. Channels were activated with $1 \mathrm{mM} \mathrm{MgATP} / 75 \mathrm{nM}$ PKA (Sigma-Aldrich), and recordings filtered at $500 \mathrm{~Hz}$ for analysis and display.

Thermal stability. Thermal stability of P67L CFTR was evaluated by inside-out patch analysis (14). Patch pipettes were pulled from Corning 8161 glass to tip resistances of $1-2 \mathrm{~m} \Omega$ (macroscopic recordings) or 9-12 $\mathrm{m} \Omega$ (unitary current recording). CFTR channels were activated following patch excision by exposure of the cytoplasmic face of the patch to the catalytic subunit of PKA (110 units/ml) and MgATP (1.5 $\mathrm{mM})$. CFTR currents were recorded in symmetrical solutions containing $140 \mathrm{mM}$ N-methyl-D-glucamine$\mathrm{Cl}, 3 \mathrm{mM} \mathrm{MgCl}$, $1 \mathrm{mM}$ EGTA, and $10 \mathrm{mM}$ TES (2-[[1,3-dihydroxy-2-(hydroxymethyl)propan-2-yl]amino] ethanesulfonic acid; $\mathrm{pH}$ 7.3). Macroscopic currents were evoked using a ramp protocol from -80 to +80 $\mathrm{mV}$ with a 10 -second time period. Patches were held at $\pm 60 \mathrm{mV}$ for unitary current recordings. An automatic controller (TC-324B, PH-3 platform, Warner Instruments) was used to regulate bath temperature by a probe in the chamber (Thermalert TH-5, Physitemp Inc.); all indicated values refer to actual bath conditions. Control experiments were conducted at room temperature $\left(22^{\circ} \mathrm{C}-23^{\circ} \mathrm{C}\right)$ prior to raising the bath temperature to approximately $37^{\circ} \mathrm{C}$. Signals from macroscopic and unitary current recordings were filtered at 20 and $200 \mathrm{~Hz}$, respectively. Data acquisition and analysis were performed using pCLAMP9.2 software (Molecular Devices LLC), including $N P_{o}$ (where $\mathrm{N}$ equals number of channels and $P_{o}$ equals open probability) and frequency of openings (openings/sec-patch).

Statistics. Data were summarized as mean \pm SEM. Replicates were treated as statistically independent in light of the fact that no comparisons were made across subjects or cell lines for any of the 3 patient samples studied: P67L/F508del, F508del/F508del, or F508del/F508del. Findings thus apply only to a specific cell line or subject evaluated, with 2-sample $t$ tests used to assess statistical significance of observed differences in mean response across two conditions applied to a particular cell line or subject. No pooled patient data or subject-to-subject comparisons were analyzed (i.e., all comparisons involve only two conditions in the same individual). The findings, therefore, should not be taken to suggest that all primary airway cells encoding P67L or F508del CFTR would behave similarly. $P$ values of less than 0.05 were considered significant.

Study approval. The studies conducted for this paper were deemed "not human subject research" by the University of Alabama at Birmingham Institutional Review Board. All cells and cell models were obtained under regulatory supervision and fully deidentified. 


\section{Author contributions}

Experiments were conducted and designed by CMS, WW, HW, CMM, WJC, and JSH, who also performed data acquisition/analysis. Reagents were furnished by KLK, JFC, JSH, MM, and EJS. Data was further analyzed and the manuscript written by CMS, JFC, JSH, KLK, and EJS.

\section{Acknowledgments}

This work was supported by grants from the Cystic Fibrosis Foundation Therapeutics (SORSCH13XX0), the NIH (P30 DK072482), and the National Center for Advancing Translational Sciences of NIH (under UL1TR000454). The content is solely the responsibility of the authors and does not necessarily represent the official views of the NIH. The authors wish to thank J. Tindall for help preparing the manuscript.

Address correspondence to: Eric J. Sorscher, 1760 Haygood Drive, Suite 280, Atlanta, Georgia 30322, USA. Phone: 404.727.3293; E-mail: esorscher@emory.edu.

1. Stoltz DA, Meyerholz DK, Welsh MJ. Origins of cystic fibrosis lung disease. N Engl J Med. 2015;372(16):1574-1575.

2. Ramsey BW, et al. Future directions in early cystic fibrosis lung disease research: an NHLBI workshop report. Am J Respir Crit Care Med. 2012;185(8):887-892.

3. Sorscher EJ. Cystic Fibrosis. In: Kasper D, Fauci A, Hauser S, Longo D, Jameson J, Loscalzo J, eds. Harrison's Principles of Internal Medicine. 19th ed. New York, New York, USA: McGraw-Hill Education/Medical, 2015:1697-1707.

4. Mendoza JL, et al. Requirements for efficient correction of $\triangle$ F508 CFTR revealed by analyses of evolved sequences. Cell. 2012;148(1-2):164-174.

5. Rabeh WM, et al. Correction of both NBD1 energetics and domain interface is required to restore $\triangle F 508$ CFTR folding and function. Cell. 2012;148(1-2):150-163.

6. Okiyoneda T, et al. Peripheral protein quality control removes unfolded CFTR from the plasma membrane. Science. 2010;329(5993):805-810.

7. Dalemans W, et al. Altered chloride ion channel kinetics associated with the delta F508 cystic fibrosis mutation. Nature. 1991;354(6354):526-528.

8. Gilfillan A, et al. P67L: a cystic fibrosis allele with mild effects found at high frequency in the Scottish population. J Med Genet. 1998;35(2):122-125.

9. Yousef S, Solomon GM, Brody A, Rowe SM, Colin AA. Improved clinical and radiographic outcomes after treatment with ivacaftor in a young adult with cystic fibrosis with the P67L CFTR mutation. Chest. 2015;147(3):e79-e82.

10. Jurkuvenaite A, et al. Mutations in the amino terminus of the cystic fibrosis transmembrane conductance regulator enhance endocytosis. J Biol Chem. 2006;281(6):3329-3334

11. Fu L, et al. $\triangle$ F508 CFTR surface stability is regulated by DAB2 and CHIP-mediated ubiquitination in post-endocytic compartments. PLoS One. 2015;10(4): $\mathrm{e} 0123131$.

12. Ren HY, et al. VX-809 corrects folding defects in cystic fibrosis transmembrane conductance regulator protein through action on membrane-spanning domain 1. Mol Biol Cell. 2013;24(19):3016-3024.

13. Cholon DM, O'Neal WK, Randell SH, Riordan JR, Gentzsch M. Modulation of endocytic trafficking and apical stability of CFTR in primary human airway epithelial cultures. Am J Physiol Lung Cell Mol Physiol. 2010;298(3):L304-L314.

14. Wang W, Okeyo GO, Tao B, Hong JS, Kirk KL. Thermally unstable gating of the most common cystic fibrosis mutant channel ( $\triangle$ F508): "rescue" by suppressor mutations in nucleotide binding domain 1 and by constitutive mutations in the cytosolic loops. J Biol Chem. 2011;286(49):41937-41948.

15. Molinski SV, et al. Genetic, cell biological, and clinical interrogation of the CFTR mutation c.3700 A>G (p.Ile1234Val) informs strategies for future medical intervention. Genet Med. 2014;16(8):625-632.

16. Rowe SM, Miller S, Sorscher EJ. Cystic fibrosis. N Engl J Med. 2005;352(19):1992-2001.

17. Hong JS, Sorscher EJ, Plyler ZE, Keiles S. Defining allelic heterogeneity among clinically important mutations in CFTR. Pediatr Pulmonol Suppl. 2014;38:Abst 170.

18. Kraus C, Reis A, Naehrlich L, Dötsch J, Korbmacher C, Rauh R. Functional characterization of a novel CFTR mutation P67S identified in a patient with atypical cystic fibrosis. Cell Physiol Biochem. 2007;19(5-6):239-248

19. Menon D, Clark D, Stafinski T. Reimbursement of Drugs for Rare Diseases through the Public Healthcare System in Canada: Where Are We Now?. Healthc Policy. 2015;11(1):15-32.

20. Evans J, Swart M, Soko N, Wonkam A, Huzair F, Dandara C. A Global Health Diagnostic for Personalized Medicine in Resource-Constrained World Settings: A Simple PCR-RFLP Method for Genotyping CYP2B6 g.15582C >T and Science and Policy Relevance for Optimal Use of Antiretroviral Drug Efavirenz. OMICS. 2015;19(6):332-338.

21. Nardini C. The ethics of clinical trials. Ecancermedicalscience. 2014;8:387.

22. Gammie T, Lu CY, Babar ZU. Access to Orphan Drugs: A Comprehensive Review of Legislations, Regulations and Policies in 35 Countries. PLoS One. 2015;10(10):e0140002.

23. Jabbour E, Makenbaeva D, Lingohr-Smith M, Lin J. Impact of genetic mutations and health plan access to therapies on treatment response and drug costs related to tyrosine kinase inhibitor treatment among patients with chronic myelogenous leukemia [published online ahead of print November 17, 2015]. Am J Clin Oncol. doi:10.1097/COC.0000000000000252.

24. Lammers P, Criscitiello C, Curigliano G, Jacobs I. Barriers to the Use of Trastuzumab for HER2+ Breast Cancer and the Potential Impact of Biosimilars: A Physician Survey in the United States and Emerging Markets. Pharmaceuticals (Basel). 2014;7(9):943-953. 
25. Van Goor F, Yu H, Burton B, Hoffman BJ. Effect of ivacaftor on CFTR forms with missense mutations associated with defects in protein processing or function. J Cyst Fibros. 2014;13(1):29-36.

26. Zomer-van Ommen DD, et al. Limited premature termination codon suppression by read-through agents in cystic fibrosis intestinal organoids. J Cyst Fibros. 2016;15(2):158-162.

27. Sosnay PR, et al. Defining the disease liability of variants in the cystic fibrosis transmembrane conductance regulator gene. Nat Genet. 2013;45(10):1160-1167.

28. Varga K, et al. Enhanced cell-surface stability of rescued DeltaF508 cystic fibrosis transmembrane conductance regulator (CFTR) by pharmacological chaperones. Biochem J. 2008;410(3):555-564.

29. Wang W, Roessler BC, Kirk KL. An electrostatic interaction at the tetrahelix bundle promotes phosphorylation-dependent cystic fibrosis transmembrane conductance regulator (CFTR) channel opening. J Biol Chem. 2014;289(44):30364-30378. 